\title{
COMPOSIÇAO DE ÁCIDOS GRAXOS DOS CORTES COMERCIAIS DE CAPIVARA (Hydrochaeris hydrochaeris L. 1766)
}

\author{
Fat acids composition of the capybara (Hydrochaeris hydrochaeris L. 1766) commercial cuts
}

\author{
Maria Cristina Bressan ${ }^{1}$, Sandra H. I. Oda ${ }^{2}$, Maria das Graças Cardoso ${ }^{1}$, Giulianna Z. Miguel' ${ }^{2}$, \\ Rilke Tadeu Fonseca de Freitas ${ }^{1}$, Josye O. Vieira ${ }^{2}$, Peter B. Faria ${ }^{2}$, Taciana V. Savian ${ }^{3}$, Sibelli P. B. Ferrão ${ }^{2}$
}

\section{RESUMO}

Com o presente trabalho teve-se como objetivo determinar o perfil de ácidos graxos (AG) em diferentes cortes comerciais da carne de capivaras. Cinco capivaras adultas foram abatidas por tiro na região temporo-occiptal, sangradas, depeladas, evisceradas, divididas em 2 meias carcaças e resfriadas a $5^{\circ} \mathrm{C}$ por 24 horas. A desossa e os cortes comerciais foram realizados às 24 horas post mortem. O percentual da gordura foi determinado segundo a AOAC (1990), e para a determinação dos AG, a gordura foi extraída segundo Folch et al. (1957) e submetida à cromatografia gasosa. Os diferentes cortes mostraram variações significati-

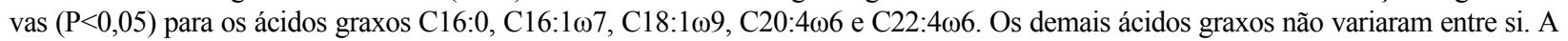
relação $\omega 3 / \omega 6$ foi de: 1:23,2; 1:10,9; 1:14,5, 1:18,7 e 1:17,3 para os cortes pernil, peito-fralda, lombo, paleta e carré, respectivamente.

Termos para indexação: Animais silvestres, capivara, gorduras, ácidos graxos.

\begin{abstract}
The present work had as objective to determine the fat acids (FA) profile in different commercial cuts of capybara meat. A total of five adults were slaughtered by shot in the temporo-occiptal region, bled, taken the skin out, eviscerated, divided in two half carcass and cooled at $5^{\circ} \mathrm{C}$ for $24 \mathrm{~h}$. The taking out of the bone and the preparation of the commercial cuts were carried out at $24 \mathrm{~h}$ post mortem. The fat percentage was determined as AOAC (1990) and for the determination of FA, extraction was performed as Folch et al. (1957), followed by gas chromatography analysis. The different cuts showed significant variations $(\mathrm{P}<0,05)$ for the $\mathrm{C} 16: 0, \mathrm{C} 16: 1 \omega 7, \mathrm{C} 18: 1 \omega 9, \mathrm{C} 20: 4 \omega 6$ and $\mathrm{C} 22: 4 \omega 6$ fat acids. The others fat acids did not differ among them. The ration $\omega 3 / \omega 6$ was of 1:23.2, 1:10.9, 1:14.5, 1:18.7 and 1:17.3 for the hind leg, chest - plati, loin, palette and carré cuts, respectively.
\end{abstract}

Index terms: Wild animals, capibara, fats, fat acid.

(Recebido para publicação em 24 de abril de 2003 e aprovado em 26 de agosto de 2004)

\section{INTRODUÇÃO}

A utilização racional ou sustentável da fauna silvestre é um processo benéfico, que pode resultar em vantagens econômicas, sociais e, ao mesmo tempo, proteger as espécies silvestres da extinção (OJASTI, 1973; ALHO, 1986; HERRERA, 1999).

A fauna de mamíferos, com sua diversidade de espécies, embora seja susceptível à perturbação, é viável para a utilização econômica, mas requer um plano de manejo e criação (ALHO, 1986). Entre as propostas de utilização da fauna silvestre de forma racional, estão: a criação dos animais em cativeiro, a implantação da caça esportiva em fazendas com criatórios de animais silvestres, a implementação de um plano de manejo extensivo, o turismo ecológico, a exploração integrada de animais silvestres com o gado e o aproveitamento de terrenos marginais. Os animais silvestres podem se transformar em fontes reno- váveis de produtos de grande rentabilidade, contribuindo para a produção de alimentos e concorrendo, em custo de produção, com os animais domésticos. Considerando essas premissas, o IBAMA (1997) homologou a Portaria número 118 (15 de outubro de 1997), que normaliza o funcionamento de criadouros de animais da fauna silvestre brasileira com fins econômicos e industriais.

Os animais silvestres produzem carne com teores reduzidos de lipídeos totais e colesterol, além de apresentarem altas proporções de ácidos graxos poliinsaturados, quando comparados aos animais domésticos (CRAWFORD et al., 1976; SINCLAIR et al., 1982; NAUGHTON et al., 1986; SINCLAIR e O'DEA, 1990). Esses aspectos nutricionais observados em animais silvestres são desejáveis, quando considerado que o conjunto de atitudes do homem moderno em relação aos hábitos de vida, tais como vida sedentária, estresse e in-

1. Professoras dos Programas de Pós-graduação da Universidade Federal de Lavras/UFLA - Caixa Postal 3037 - $37200-000$ - Lavras, MG.

2. Alunos do Programa de Pós-graduação em Ciência dos Alimentos/UFLA.

3. Aluna do Programa de Pós-graduação em Estatística da Universidade Federal de Lavras/UFLA. 
gestão elevada de alimentos ricos em gorduras, é associado a quadros de obesidade, hipertensão, hipercolesterolemia e problemas cardiocirculatórios. Além disso, os profissionais da área de saúde relacionam a elevada incidência de enfermidades cardiocirculatórias ao consumo de carne vermelha (WOOD, 1990). Essa posição tem sido adotada nas últimas décadas, ocasionando uma redução na ingestão de carne, um importante componente da dieta, que fornece aminoácidos e ácidos graxos essenciais, vitaminas e sais minerais (FORREST et al., 1979; PARDI et al., 1993).

Atualmente, o mercado consumidor de carne tem se mostrado bastante receptivo ao consumo de carne de animais silvestres e exóticos. Apesar da existência de uma demanda pela carne de capivara nos grandes centros e da possibilidade de abertura de novos mercados (mercado externo), os estudos da composição química da carne de capivara e aspectos nutricionais são escassos e trabalhos que caracterizam a composição dos possíveis cortes comerciais são restritos. Assim, objetivouse com o presente trabalho analisar a composição de ácidos graxos nos diferentes cortes comerciais da carne de capivaras.

\section{MATERIAL E MÉTODOS}

No presente trabalho, foram utilizados 5 animais adultos com com peso vivo médio de $63,8 \mathrm{~kg}$, machos e fêmeas, provenientes de cativeiro (baias individuais), alimentados com forragens (napier) e ração à base de milho e farelo de soja, com $15 \%$ de proteína bruta e $3375 \mathrm{kcal} / \mathrm{kg}$ de energia digerível, formulada de acordo com as exigências nutricionais recomendadas para suínos em terminação (ROSTAGNO et al., 2000).

Os animais foram abatidos por tiro na região temporo-ocipital, seguindo-se as seguintes etapas: a) sangria por secção das artérias carótidas e veias jugulares; b) retirada dos pêlos por queima, seguida de lavagem e raspagem externa; c) evisceração; d) divisão da carcaça em meias carcaças; e e) resfriamento das meias carcaças a $5^{\circ} \mathrm{C}$ por 24 horas.

A meia carcaça foi separada em cortes, conforme proposta descrita por Santos (1999) para ovinos, em cinco regiões anatômicas denominadas: pernil, paleta, peito/fralda (costela/fralda), carré (costeleta), lombo e pescoço, conforme descrito a seguir e mostrado na Figura 1:

Paleta - as regiões anatômicas que compreenderam esse corte foram o cíngulo escapular, braço e antebraço. A base óssea foi formada pela escápula, úmero, rádio e ossos do carpo. O corte foi obtido mediante séc- ção da região axilar e dos músculos que unem a escápula e o úmero na parte ventral do tórax. Depois, foi contornada a escápula, seccionando os músculos braquiocefálico, omo transversal, trapézio cervical e serrato cervical, pela parte superior, e trapézio torácico e rombóides pela parte posterior do tronco;

Pernil (perna) - compreendeu a região sacral e os seguintes segmentos anatômicos do membro pélvico: cíngulo pélvico e perna. A base óssea foi formada pelo tarso, tíbia, fêmur, ísquio, púbis e íleo. O corte foi obtido pela secção na região da articulação da última vértebra lombar e primeira sacral e na região da posição inicial dos ossos do tarso;

Lombo - a base óssea desse corte compreendeu da primeira à última vértebra lombar (6 ou 7 vértebras). Para obtenção do corte, foi realizada, inicialmente, a secção entre a última vértebra torácica e primeira lombar, seguida de uma outra secção entre a última lombar e a primeira sacral;

Carré (costeleta) - compreendeu a região localizada entre a $1^{\mathrm{a}}$ e a $13^{\mathrm{a}}$ vértebra torácica, junto com aproximadamente $1 / 3$ dorsal do corpo das costelas correspondentes; e

Peito/fralda (costela/fralda) - esse corte compreendeu a região anatômica da parede abdominal e $2 / 3$ da região ventral torácica. Sua base óssea foi metade correspondente do esterno cortado sagitalmente, aproximadamente $2 / 3$ ventrais das oito primeiras costelas e terço ventral das cinco restantes.

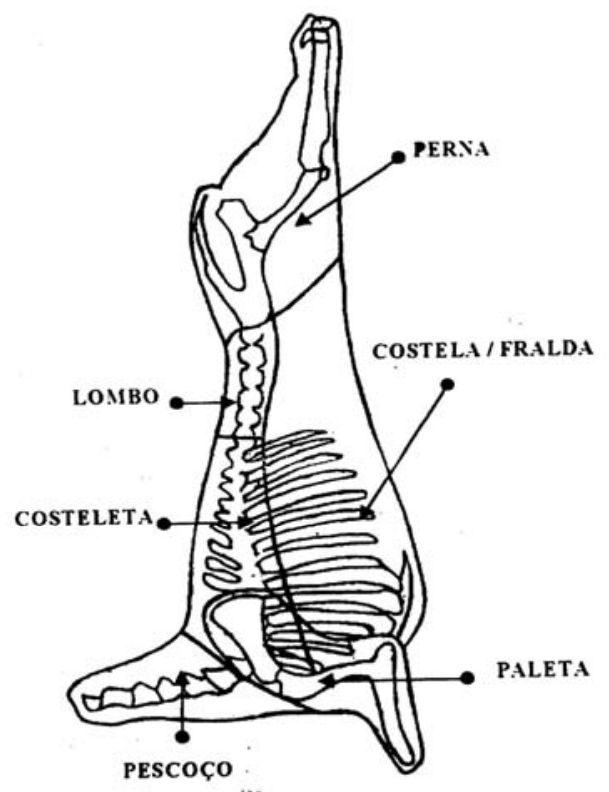

FIGURA 1 - Cortes realizados na $1 / 2$ carcaça esquerda. 
Os cortes, após a desossa, foram acondicionados em sacos de polietileno e mantidos em freezer a $-12^{\circ} \mathrm{C}$. Desses cortes, os músculos foram dissecados (sem a gordura subcutânea e o tecido conectivo visível) e homogeneizados. Amostras de 50 gramas foram separadas, identificadas, congeladas a $-20^{\circ} \mathrm{C}$ e para proceder às análises, as amostras foram descongeladas a $4^{\circ} \mathrm{C}$.

Para a realização das análises de ácidos graxos, os lipídeos foram extraídos conforme metodologia de Folch et al. (1957), adaptada para amostras de 5 gramas, que foi homogeneizada em $50 \mathrm{~mL}$ de clorofórmio/metanol (2:1). A amostra homogeneizada foi filtrada para funil de separação de $250 \mathrm{~mL}$, permanecendo em repouso por 2 horas, para a separação física. A fração apolar do homogeneizado, contendo lipídios e clorofórmio, foi recolhida e a fração polar foi descartada. A fração apolar foi submetida à nova separação por 12 horas e, dessa segunda separação, a fração apolar foi recolhida em balão volumétrico, sendo adicionado clorofórmio até completar $50 \mathrm{~mL}$. Desse extrato, foram retirados $5 \mathrm{~mL}$ para a determinação do perfil de ácidos graxos.

Essas amostras foram inicialmente saponificadas com hidróxido de sódio com metanol 0,5 M e metiladas com solução de cloreto de amônia, metanol e ácido sulfúrico, segundo Hartman e Lago (1973). Após a metilação, $5 \mathrm{~mL}$ de hexano foram adicionados à amostra $\mathrm{e}$ submetidas a agitação por 10 segundos. Do sobrenadante, foi retirada uma alíquota de $3 \mathrm{~mL}$, que foi concentrada com nitrogênio gasoso, ressuspendida em $100 \mu \mathrm{L}$ de hexano e, para a injeção, foi tomada uma alíquota de $1 \mu \mathrm{L}$.

As amostras foram submetidas à cromatografia gasosa e injetadas manualmente em cromatógrafo a gás (marca Shimadzu, modelo GC-17A), equipado com detector de ionização de chama, injetor split na razão de 1:5, coluna capilar de polietileno-glicol DB-Wax (30 m; 0,25 $\mathrm{mm} ; 0,25 \mu \mathrm{m})$ e acoplado a um software desenvolvido pela Shimadzu. As condições cromatográficas foram: temperatura inicial da coluna: $180^{\circ} \mathrm{C}$ por 10 minutos, aumentada a uma taxa de $5^{\circ} \mathrm{C} /$ minutos até a temperatura final da coluna de $240^{\circ} \mathrm{C}$, permanecendo nessa temperatura por $18 \mathrm{mi}-$ nutos; temperatura do injetor: $230^{\circ} \mathrm{C}$; e temperatura do detector: $250^{\circ} \mathrm{C}$. O gás de arraste utilizado foi o nitrogênio em fluxo de $01 \mathrm{~mL} /$ minuto.

Os diferentes ácidos graxos foram identificados por comparação aos tempos de retenção apresentados pelo padrão cromatográfico (Pufa 2, Sigma-Aldrich), constituído por uma mistura de 14 ácidos graxos. A quantificação dos ácidos graxos foi realizada por normalização.

$\mathrm{O}$ delineamento experimental foi em blocos casualizados, sendo considerado bloco o animal, com 5 tratamentos (5 diferentes cortes comerciais) e 5 repetições, sen- do o animal a unidade experimental. Para as análises dos dados obtidos, foi utilizado o pacote computacional Sisvar (FERREIRA, 2000) e o modelo estatístico seguido foi:

$$
\mathrm{Y}_{\mathrm{ij}}=\mu+\mathrm{C}_{\mathrm{i}}+\mathrm{A}_{\mathrm{j}}+\mathrm{e}_{\mathrm{ij}} \text {, em que: }
$$

$\mathrm{Y}_{\mathrm{ij}}=$ observação do corte comercial i, no animal j; $\mu=$ é a constante associada a todas as observações; $C_{i}=$ efeito dos cortes comerciais $i$, sendo $i=1,2,3,4$ e 5 ; $A_{j}=$ efeito do animal $j$ (bloco), para $j=1,2,3,4$ e 5; $e_{i j}=$ é o erro experimental associado à observação $Y_{i j}$, que, por hipótese, tem distribuição normal independentente, distribuído com média zero e variância $\sigma^{2}$.

Quando a análise de variância identificou diferença significativa entre os cortes para cada variável analisada, as médias foram submetidas ao teste de ScottKnott ao nível de probabilidade inferior a $5 \%(\mathrm{P}<0,05)$.

\section{RESULTADOS E DISCUSSÃO}

Os resultados médios para lipídios totais (LT) nos diferentes cortes comerciais de capivara são apresentados na Tabela 1. Embora não tenha sido demonstrada diferença significativa entre cortes, essa variação foi de 0,36 a $1,25 \%$. Considerando a média geral de LT de $0,85 \%$ ( 850 $\mathrm{mg} / 100 \mathrm{~g}$ ), resultados semelhantes em capivaras foram descritos por Jardim (2001) no músculo longissimus dorsi (LD) (lombo), com média de $0,82 \mathrm{~g} / 100 \mathrm{~g}$, e por Roça et al. (1996), em copa, com média de 0,91 g/100g. Entretanto, Saldanha et al. (2002) descreveram valores mais elevados de lipídios totais nos cortes pernil e paleta, com média de $1,6 \mathrm{~g} / 100 \mathrm{~g}$. Em espécies convencionais, médias mais elevadas foram descritas. Bragagnolo (1997) cita média de 2,5 g/100g em contrafilé de bovinos (Nelore) e Bragagnolo e Rodriguez-Amaya (2002) relataram média de $5 \mathrm{~g} / 100 \mathrm{~g}$ em paleta de suíno. Em ovinos, Souza et al. (2002) encontraram, no bíceps femoris, médias de 1,48, 2,07, 3,14 e 3,79 g/100g em animais de $15,25,35$ e $45 \mathrm{~kg}$, respectivamente; e Bonagurio (2001) descreveu valores entre 1 e 3,5 $\mathrm{g} / 100 \mathrm{~g}$ no mesmo músculo em animais de 15 a 45 $\mathrm{kg}$. Vários fatores influenciam o percentual de gordura na carne, tais como sexo (FORREST et al., 1979; SOUZA et al., 2002), idade de abate e grupamento genético (FORREST et al., 1979; BONAGURIO, 2001; SOUZA et al., 2002). Entretanto, independente desses aspectos, a carne de capivara de animais adultos mostra-se com percentual de gordura mais baixo do que as demais carnes convencionais usadas na dieta humana abatidas em fase de terminação, sendo também mais baixo do que em ovinos abatidos com $15 \mathrm{~kg}$ de peso vivo, descrito 
por Souza et al. (2002), com média de 1,48 g/100g no músculo bíceps femoris e por Bonagurio (2001), com média superior a $1 \mathrm{~g} / 100 \mathrm{~g}$ de lipídios totais. Essa quantidade de gordura assemelha-se às médias verificadas em canguru (SINCLAIR e O'DEA, 1990).

Os resultados médios de AG saturados, monoinsaturados, poliinsaturados, total de ácidos graxos (AG) do tipo $\omega 3$ e $\omega 6$ e a relação $\omega 3 / \omega 6$ são mostrados na Tabela 1 .

Pela análise estatística, constatou-se que entre cortes houve variação significativa $(\mathrm{P}<0,05)$ para os $\mathrm{AG}$

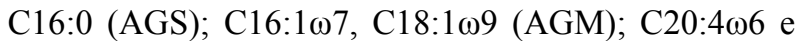
$\mathrm{C} 22: 4 \omega 6$ (AGP). Os demais AG mostraram resultados semelhantes entre os cortes.

Em termos quantitativos (\%), os $\mathrm{AG}$ que apresentaram maior proporção, em ordem decrescente, fo-

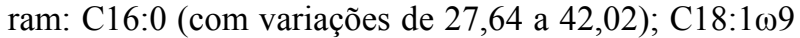

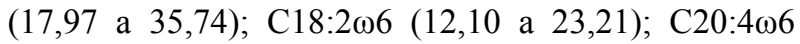

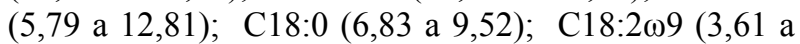
6,01); C14:0 (1,66 a 3,04); C18:3 13 (1,00 a 1,27);

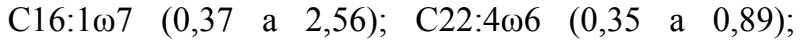

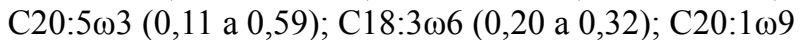
$(0,18$ a 0,29$)$ e $\mathrm{C} 22: 6 \omega 6(0,10$ a 0,24$)$.

TABELA 1 - Lipídios totais e composição de ácidos graxos em cortes de capivara.

\begin{tabular}{|c|c|c|c|c|c|}
\hline \multirow[b]{2}{*}{ AG } & \multicolumn{5}{|c|}{ Cortes Comerciais } \\
\hline & $\begin{array}{c}\text { Pernil } \\
\text { Média } \pm \text { ep }\end{array}$ & $\begin{array}{l}\text { Peito-Fralda } \\
\text { Média } \pm \text { ep }\end{array}$ & $\begin{array}{c}\text { Lombo } \\
\text { Média } \pm \text { ep }\end{array}$ & $\begin{array}{c}\text { Paleta } \\
\text { Média } \pm \text { ep }\end{array}$ & $\begin{array}{c}\text { Carré } \\
\text { Média } \pm \text { ep }\end{array}$ \\
\hline LT & $0,36 \pm 0,32^{\mathrm{a}}$ & $1,25 \pm 0,32^{\mathrm{a}}$ & $0,83 \pm 0,32^{\mathrm{a}}$ & $0,60 \pm 0,32^{\mathrm{a}}$ & $1,18 \pm 0,32^{\mathrm{a}}$ \\
\hline \multicolumn{6}{|l|}{ AGS } \\
\hline C 14:0 & $1,66 \pm 0,41^{\mathrm{a}}$ & $3,04 \pm 0,40^{\mathrm{a}}$ & $1,80 \pm 0,40^{\mathrm{a}}$ & $2,17 \pm 0,40^{\mathrm{a}}$ & $2,47 \pm 0,40^{\mathrm{a}}$ \\
\hline C $16: 0$ & $27,64 \pm 2,31^{\mathrm{b}}$ & $35,23 \pm 2,31^{\mathrm{ab}}$ & $42,021 \pm 2,64^{\mathrm{a}}$ & $34,21 \pm 2,31^{\mathrm{ab}}$ & $32,07 \pm 2,31^{\mathrm{ab}}$ \\
\hline C 18:0 & $9,06 \pm 1,12^{\mathrm{a}}$ & $7,85 \pm 1,28^{\mathrm{a}}$ & $8,09 \pm 1,28^{\mathrm{a}}$ & $9,52 \pm 1,12^{\mathrm{a}}$ & $6,83 \pm 1,12^{\mathrm{a}}$ \\
\hline Total AGS & 38,36 & 46,32 & 51,90 & 45,90 & 41,37 \\
\hline \multicolumn{6}{|l|}{ AGM } \\
\hline C $16: 1 \omega 7$ & $0,37 \pm 0,36^{\mathrm{b}}$ & $2,56 \pm 0,36^{\mathrm{a}}$ & $0,91 \pm 0,36^{\mathrm{b}}$ & $1,22 \pm 0,36^{\mathrm{ab}}$ & $1,14 \pm 0,36^{a b}$ \\
\hline C $18: 1 \omega 9$ & $17,97 \pm 2,79^{b}$ & $35,74 \pm 2,79^{\mathrm{a}}$ & $29,31 \pm 2,79^{\mathrm{ab}}$ & $26,27 \pm 2,79^{\mathrm{ab}}$ & $26,95 \pm 2,79^{\mathrm{ab}}$ \\
\hline C $20: 1 \omega 9$ & $0,26 \pm 0,06^{\mathrm{a}}$ & $0,20 \pm 0,06^{\mathrm{a}}$ & $0,29 \pm 0,06^{\mathrm{a}}$ & $0,18 \pm 0,06^{\mathrm{a}}$ & $0,23 \pm 0,06^{\mathrm{a}}$ \\
\hline Total AGM & 18,60 & 38,50 & 30,51 & 27,67 & 28,32 \\
\hline \multicolumn{6}{|l|}{ AGP } \\
\hline C $18: 2 \omega 9$ & $3,61 \pm 0,65^{\mathrm{a}}$ & $4,70 \pm 0,65^{\mathrm{a}}$ & $6,01 \pm 0,65^{\mathrm{a}}$ & $4,50 \pm 0,65^{\mathrm{a}}$ & $4,28 \pm 0,65^{\mathrm{a}}$ \\
\hline C $18: 2 \omega 6$ & $23,21 \pm 2,72^{\mathrm{a}}$ & $12,10 \pm 2,72^{a}$ & $16,77 \pm 2,72^{\mathrm{a}}$ & $20,28 \pm 2,72^{\mathrm{a}}$ & $17,52 \pm 2,72^{\mathrm{a}}$ \\
\hline C $18: 3 \omega 6$ & $0,20 \pm 0,05^{\mathrm{a}}$ & $0,25 \pm 0,05^{\mathrm{a}}$ & $0,31 \pm 0,05^{\mathrm{a}}$ & $0,32 \pm 0,05^{\mathrm{a}}$ & $0,25 \pm 0,05^{\mathrm{a}}$ \\
\hline C $18: 3 \omega 3$ & $1,27 \pm 0,18^{\mathrm{a}}$ & $1,00 \pm 0,18^{\mathrm{a}}$ & $1,17 \pm 0,21^{\mathrm{a}}$ & $1,16 \pm 0,18^{\mathrm{a}}$ & $1,12 \pm 0,18^{\mathrm{a}}$ \\
\hline C $20: 4 \omega 6$ & $12,81 \pm 1,50^{\mathrm{a}}$ & $5,79 \pm 2,04^{b}$ & $11,18 \pm 1,73^{\mathrm{ab}}$ & $5,87 \pm 1,50^{\mathrm{b}}$ & $8,80 \pm 1,50^{\mathrm{ab}}$ \\
\hline C $20: 5 \omega 3$ & $0,19 \pm 0,17^{\mathrm{a}}$ & $0,59 \pm 0,44^{\mathrm{a}}$ & $0,59 \pm 0,20^{\mathrm{a}}$ & $0,11 \pm 0,20^{\mathrm{a}}$ & $0,28 \pm 0,17^{\mathrm{a}}$ \\
\hline C $22: 6 \omega 3$ & $0,14 \pm 0,01^{\mathrm{a}}$ & $0,10 \pm 0,02^{\mathrm{a}}$ & $0,24 \pm 0,02^{\mathrm{a}}$ & $0,17 \pm 0,02^{\mathrm{a}}$ & $0,17 \pm 0,01^{\mathrm{a}}$ \\
\hline C $22: 4 \omega 6$ & $0,89 \pm 0,11^{\mathrm{a}}$ & $0,35 \pm 0,12^{\mathrm{b}}$ & $0,73 \pm 0,12^{\mathrm{ab}}$ & $0,45 \pm 0,11^{\mathrm{ab}}$ & $0,55 \pm 0,1^{1 \mathrm{ab}}$ \\
\hline Total AGP & 43,32 & 24,89 & 67,51 & 32,86 & 32,97 \\
\hline Total $\omega 3$ & $1,60 \pm 0,14^{\mathrm{a}}$ & $1,69 \pm 0,14^{\mathrm{a}}$ & $2,00 \pm 0,14^{\mathrm{a}}$ & $1,44 \pm 0,14^{\mathrm{a}}$ & $1,57 \pm 0,14^{\mathrm{a}}$ \\
\hline Total $\omega 6$ & $37,11 \pm 0,94^{\mathrm{a}}$ & $18,48 \pm 0,94^{b}$ & $28,99 \pm 0,94^{\mathrm{ab}}$ & $26,92 \pm 0,94^{\mathrm{ab}}$ & $27,12 \pm 0,94^{\mathrm{ab}}$ \\
\hline$\omega 3 / \omega 6$ & 23,19 & 10,95 & 14,50 & 18,69 & 17,27 \\
\hline
\end{tabular}

${ }^{a b}$ Médias seguidas de letras diferentes diferem significativamente $(P<0,05)$

Média \pm erro-padrão de 5 amostras analisadas em duplicatas

AGS = Ácidos Graxos Saturados. AGM= Ácidos Graxos Monoinsaturados

AGP = Ácidos Graxos Poliinsaturados 
O C14:0, considerado um ácido graxo capaz de aumentar o colesterol sérico (KEY et al., 1965), apresentou uma variação de 1,66 a 3,04\% (sem diferença significativa) nos cortes avaliados. Jardim (2001), trabalhando com capivaras em idade de abate comercial, encontrou resultados médios que variam de 1,40 a 1,52\% em músculos LD; Saldanha et al. (2002) encontraram média de 1,29\% e, nessa espécie, Oda (2002) relatou médias que variaram de 3,33 a 3,93\% no músculo semimembranosus (SM). As variações médias encontradas no presente estudo encontram-se entre os valores de C14:0, citados na literatura para capivaras. Em ovinos, Kemp et al. (1981) determinaram no músculo LD percentuais de 3,0\%. Em bovinos e ovinos, valores mais elevados, variando de 3,76 a 5,40\%, foram obtidos por Onyango et al. (1998), Calles et al. (2000) e Perez et al. (2002).

O C16:0, outro AG hiperlipidêmico (KEY et al., 1965), mostrou médias com diferenças significativas $(\mathrm{P}<0,05)$ entre cortes. No lombo, foi observado valor de $42,02 \%$ e, no perfil, a menor média $(27,64 \%)$ e valores intermediários $(35,23 ; 34,21$ e $32,07 \%)$ foram encontrados nos cortes peito-fralda, paleta e carré. No presente trabalho, as médias variaram de 27,64 a 42,02\%. Jardim (2001) e Saldanha et al. (2002) relataram valores mais baixos em capivaras, os quais variaram de 13,68 a 17,64\%; entretanto, Oda (2002) cita valores de 27,34 a $31,80 \%$, que foram mais próximos aos resultados encontrados no presente trabalho. Em bovinos, os valores variam de 24,8 a $30,8 \%$ (BRAGAGNOLO e RODRIGUEZ-AMAYA, 1995; PALEARI et al., 1998).

$\mathrm{O}$ ácido graxo esteárico (C18:0) apresentou variações de 6,83 a $9,52 \%$ entre os cortes estudados. Oda (2002) e Saldanha et al. (2002) encontraram médias que variaram de 4,98 a 7,53\% no músculo SM e paleta, respectivamente, e resultados mais elevados foram reportados por Jardim (2001), cujos valores variaram de 10,76 a $11,40 \%$ no músculo LD. Embora o C18:0 seja um AG saturado de cadeia longa, ele é considerado neutro em relação às concentrações plasmáticas de colesterol, pois, após sua ingestão, é rapidamente convertido a ácido oléico (monoinsaturado) pelo organismo.

Os percentuais das áreas de pico dos AGS do presente estudo nos diferentes cortes variaram de 38,36 a 51,90\%. Na espécie em estudo, são reportados valores de 38,00 a 43,90\% (JARDIM, 2001; SALDANHA et al., 2002; ODA, 2002). Em ovinos, caprinos, perus e bovinos, são descritas variações entre 42,19 a 55,07\% (PALEARI et al., 1998; ROWE et al., 1999; RHEE et al., 2000; CALLES et al., 2000; PEREZ et al., 2002). Em espécies silvestres, foram reportados valores de
AGS de 25,4 a 45,6\% em zebras, emas e avestruzes (ONYANGO et al., 1998; SALES et al., 1999). Observando os dados da literatura, observa-se que para o total de AGS existe uma grande variação mesmo na espécie e isso pode ser atribuído a inúmeros fatores, tais como: dieta (ROSA, 1999), local de coleta da amostra, músculos utilizados, preparo da amostra (com ou sem a retirada da gordura aparente). Forrest et al. (1979) citaram que gorduras de depósito mostram uma maior quantidade de AG saturados (do que gorduras extraídas do tecido cárneo magro), metodologia de determinação e quantificação, bem como o número de AGS determinados (alguns autores determinam, além do C14:0; C16:0 e C18:0, os AG C10:0, C12:0 e C17:0).

As médias de $\mathrm{C} 16: 1 \omega 7$ (AG palmitoléico) foram diferentes $(\mathrm{P}<0,01)$ entre cortes. $\mathrm{O}$ corte peito-fralda apresentou média mais elevada $(2,56 \%)$, do que o pernil $(0,37 \%)$ e o lombo $(0,91 \%)$, entretanto, os cortes de paleta e carré mostraram valores intermediários $(1,22$ e $1,14 \%$, respectivamente). Valores próximos (1,24 a $2,54 \%$ ) foram relatados por Oda (2002) no músculo SM (pernil). Porém, Jardim (2001) cita valores mais baixos (com variação de 0,54 a $0,76 \%$ ) no músculo LD (lombo).

O AG oléico (C18:1 19$)$ tem uma importante participação nos processos fisiológicos, tais como a manutenção da fluidez das membranas e o efeito hipocolesterolêmico (SPECTOR, 1999), e é uma fonte preferencial de energia metabolizável no organismo humano (WALDMAN et al., 1965). Bonanome e Grundy (1988) verificaram que dietas ricas em C18:1 199 proporcionaram redução nos teores de colesterol total plasmático, de LDL colesterol e na relação LDL/HDL colesterol, demonstrando o efeito positivo de dietas com elevados percentuais de AG oléico na alimentação humana. Esse

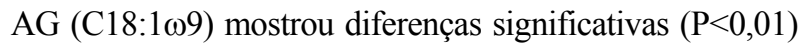
entre as médias dos cortes estudados. Valores mais elevados foram observados no peito e fralda $(35,74 \%)$, mais baixos no pernil $(17,29 \%)$ e com valores intermediários no lombo, paleta e carré $(29,31,26,27$ e $26,95 \%$, respectivamente). A variação média foi de 17,29 a 35,74\%. Valores semelhantes foram reportados em capivaras por Oda (2002), com variação de 25,73 a $28,05 \%$, e superiores aos valores descritos por Jardim (2001), com variações de 9,08 a $11,66 \%$. Entretanto, esses resultados mostraram-se inferiores ao observados em ovinos (30,73 a 45,19\%) (PALEARI et al., 1998; ROWE et al., 1999; RHEE et al., 2000; CALLES et al., 2000; PEREZ et al., 2002) e em frangos (39,8\%) (SALES et al., 1999).

As variações dos resultados totais de AGM foram de 18 a 38,5\%. Em capivaras, Saldanha et al. (2002) e Jar- 
$\operatorname{dim}$ (2001) relataram valores totais de 11,70 a $25,05 \%$ e Oda (2002), valores de 27,17 a 30,83\%.

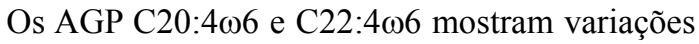
significativas $(\mathrm{P}<0,05)$ quando comparados aos diferen-

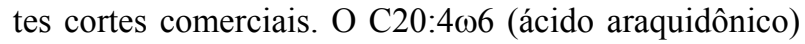
mostrou média superior no corte pernil $(12,81 \%)$ quando comparado ao corte peito-fralda (5,79\%); entretanto, esses valores foram semelhantes às médias verificadas nos cortes lombo $(11,18 \%)$, paleta $(5,87)$ e carré $(8,80 \%)$. Comportamento semelhante foi verificado para o $\mathrm{C} 22: 4 \omega 6$, com média superior no pernil $(0,89 \%$, em relação ao corte peito-fralda $(0,35 \%)$, e esses resultados foram estaticamente semelhante as médias no lombo $(0,73 \%)$, patela $(0,45 \%)$ e carré $(0,55 \%)$.

Os AG essenciais linoléico, linolênico e araquidônico, para a sua utilização no organismo de mamíferos, devem ser obtidos a partir da dieta, pois a conversão de um $A G$ de uma família $\omega$ em outra não ocorre. $O$ AGP linoléico (C18:2 $\omega 6$ ), da família $\omega 6$, pode dar ori-

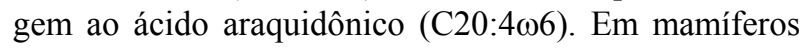
em crescimento e até a meia idade, o linoléico pode ser

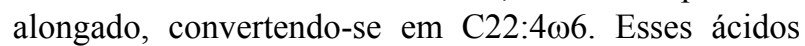
graxos essenciais estão associados à síntese de eicosanóides, que são substâncias modeladoras de muitas funções vitais, participando de processos secretórios, digestivos, reprodutivos, imunológicos e circulatórios (MANCINI-FILHO e CHEMIN, 1996). Além disso, o AG araquidônico encontra-se presente nas membranas celulares e representa de 5 a $15 \%$ dos AG que estão esterificados nos fosforolipídeos (CRAWFORD et al., 1976). Considerando as diferentes concentrações

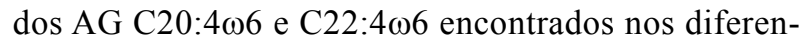
tes cortes, é possível supor que: a) os músculos que compõem os diferentes cortes apresentam diferentes quantidades desses AG na composição de membranas, resultado possivelmente da diferença na composição do músculo em relação aos tipos de fibras, que são do tipo glicolíticas, glicolítica-oxidativa ou oxidativa (conhecidas como fibras brancas, fibras intermediárias ou fibras vermelhas) (ALASNIER, 1996); b) os músculos que compõem os diferentes cortes apresentam diferentes requerimentos em AG da família $\omega 6$, considerando que a composição dos músculos difere em relação ao tipo de fibra, que apresenta funções orgânicas e metabolismo diferente; e c) os músculos que compõem os diferentes cortes apresentam diferente composição de membranas e diferentes requerimentos em AG da série $\omega 6$.

Os demais AG da série $\omega 6$ não apresentaram variações entre cortes. $\mathrm{E}$ desses $\mathrm{AG}$ determinados, o C18:2 06 (linoléico) foi o AG mais abundante com varia- ções de 12,10 a 23,21\%. Em capivaras, Jardim (2001) e Oda (2002) relataram valores entre 17,04 a $21,34 \%$ e Saldanha et al. (2002) relataram médias mais baixas de $15,11 \%$. Em espécies domésticas, Perez et al. (2002) reporta médias de 4,42 a 10,32\% em ovinos no músculo LD; Bragagnolo e Rodriguez-Amaya (1995) citam valores de 5 e 9\% em contrafilé bovino (raça Nelore) e em lombo suíno, respectivamente; e Garcia e Casal (1999) encontraram médias de $24,5 \%$ em peito de frango.

Com relação aos AG de família $\omega 3$ (sem diferença significativa entre cortes), o $\mathrm{C} 18: 3 \omega 3$ (ácido $\alpha$ linolênico, precursor dos AG C20:5 13 e C22:6 13 ) foi o AG mais abundante, com variações de 1 a $1,27 \%$. Em capivaras, valores mais elevados $(4,39$ a $5,85 \%)$ foram relatados por Oda (2002) e mais baixos $(0,24$ a $0,47 \%)$ por Jardim (2002). Entretanto, Saldanha et al. (2002) encontraram médias de $1,73 \%$, semelhante aos resultados do presente trabalho. A diferença entre autores pode ser atribuída às diferentes dietas ingeridas pelos animais monogástricos (ROSA, 2000).

O total de AGP variou de 24,89 a 43,32\%. Em capivaras, são descritas variações de 25,36 a $35,41 \%$ (JARDIM, 2001; SALDANHA et al. 2002; ODA, 2002). Em espécies de açougue - ovinos, caprinos e bovinos - são relatadas médias de 5,36 a $12,54 \%$ (BRAGAGNOLO e RODRIGUEZ-AMAYA, 1995; ROWE et al., 1999; RHEE et al., 1999; PEREZ et al., 2002). Essas variações entre animais podem ser atribuídas ao tipo de alimento e à fisiologia digestiva.

Os valores médios para $\omega 3$ e $\omega 6$ variaram de 1,44 a $2,0 \%$ e 18,48 a $37,11 \%$, respectivamente. Esses resultados são semelhantes aos de Oda (2002) para o total de $\omega 6(25,95 \%)$, mas inferiores para $\omega 3(5,73 \%)$. Saldanha et al. (2002) citaram variações para AG $\omega 3$ de 0,8 a 3,3\% no lombo e paleta de capivaras. Segundo recomendações da FAO (1994), a relação de AG da família $\omega 6$ e $\omega 3$ deve ser de 3-4:1. Essas relações foram calculadas para os cortes comerciais, sendo o menor valor encontrado de 10,95 para o peito-fralda e o maior de 23,19 para a paleta.

\section{CONCLUSÕES}

Os diferentes cortes apresentam diferentes per-

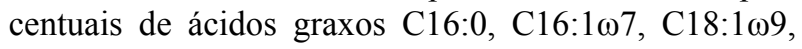

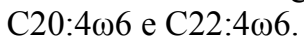

\section{AGRADECIMENTOS}

Ao CNPq e à FAPEMIG, pela concessão de bolsa de mestrado, e à Empresa Pró-Fauna Assessoria e Comércio Ltda, pela doação de amostras e apoio logístico. 


\section{REFERÊNCIAS BIBLIOGRÁFICAS}

ALASNIER, C.; REMINGN, H.; GANDEMER, G. Lipids characteristics associated with oxidative and glycolytic fibres in rabbit muscle. Meat Science, Oxford, v. 43, n. 3-4, p. 213-224, 1996.

ALHO, C. J. R. Criação e manejo de capivaras em pequenas propriedades rurais. Brasília: EMBRAPADDT, 1986. 48 p. (Documentos, 13).

ASSOCIATION OF OFFICIAL ANALYTICAL CHEMISTS. Official methods of analysis of the Association of Official Analytical Chemists. 15. ed. Arlington, 1990.

BONAGURIO, S. Qualidade da carne de cordeiros Santa Inês puros e mestiços com Texel abatidos com diferentes pesos. 2001. 150 p. Dissertação (Mestrado em Zootecnia) - Universidade Federal de Lavras, Lavras, 2001.

BONANOME, A. M. D.; GRUNDY, S. M. Effect of dietary stearic acid on plasma cholesterol and lipoprotein levels. New England Journal of Medicine, Boston, v. 318, n. 19, p. 1244-1247, May 1988.

BRAGAGNOLO, N. Fatores que influenciam o nível de colesterol, lipídeos totais e composição de ácidos graxos em camarão e carne. 1997. 123 f. Tese (Doutorado em Ciência de Alimentos) - Universidade de Campinas, Campinas, 1997.

BRAGAGNOLO, N.; RODRIGUEZ-AMAYA, D. B. Teores de colesterol, lipídios totais e ácidos graxos em cortes de carnes. Ciência e Tecnologia de Alimentos, Campinas, v. 22, n. 1, p. 98-104, 2002.

CALLES, J. A. E. et al. Sire variation in fatty acid composition of croobred Wagyu steers and heifers. Meat Sicense, Oxford, v. 56, n. 1, p. 23-29, Sept. 2000 .

CRAWFORD, M. A.; CASPERD, M. N.; SINCLAIR, A. J. The long chain metabolites of linoleic and linolenic acids and liver and brain in herbivoros and carnivores. Comparative Biochenistry and Physiology - fart B, Elmsford, v. 54B, p. 395-401, 1976.

FAO/WHO. Report of a joint expert consulation: fats and oils in human nutrition. Food and Nutrition Paper, Rome, v. 57, n. 1, p. 49-55, 1994.
FERREIRA, D. F. Análises estatísticas por meio do Sisvar para Windows: versão 4.0. In: REUNIÃO ANUAL DA REGIÃO BRASILEIRA DA SOCIEDADE INTERNACIONAL DE BIOMETRIA, 45., 2000, São Carlos. Anais... São Carlos: UFSCAR, 2000. p. 255-258.

FOLCH, J.; LEES, M.; SLOANE-STANLEY, G. H. A simple method for the isolation and purification of total lipids from animal tissue. Journal of Biological Chemistry, Baltimore, n. 226, p. 497-509, 1957.

FORREST, J. C. et al. Fundamentos de ciência de la carne. Zaragoza: Acríbia, 1979. 364 p.

GARCIA, P. T.; CASAL, J. J. Contribution of poultry lipids to corrent recommendation for an optimum lipid dietary intake. In: INTERNATIONAL CONGRESS OF MEAT SCIENCE AND TECHNOLOGY, 45, 1999, Yokohama. Anais... Yokohama: ICOMST, 1999.

HARTMAN, L.; LAGO, R. C. Rapid preparation of fatty acid methyl esters. Laboratory Practice, London, v. 494, n. 22, p. 475-476, Jul. 1973.

HERRERA, E. A. Comportamiento, conservación y manejo de fauna silvestre: el caso del capibara en Venezuela. Etología, [S.1.], v. 7, p. 41-46, 1999.

INSTITUTO BRASILEIRO DO MEIO AMBIENTE E DOS RECURSOS NATURAIS RENOVÁVEIS. Portaria $\mathbf{N}^{\circ}{ }^{\circ}$ 118/97 de 15.10.97, regulamentação da criação de animais da fauna silvestre brasileira com fins econômicos e industriais. Disponível em: $<$ http://www.ibmapr.hpg.ig. com.br/Prt118-97.htm>. Acesso em: 15 jun. 2004.

JARDIM, N. S. Sexo e diferentes pesos ao abate na qualidade da carne de capivara (Hydrochaeris hydrochaeris L. 1766). 2001. 119 p. Dissertação (Mestrado em Ciência de Alimentos) - Universidade Federal de Lavras, Lavras, 2001.

KEMP, J. D. et al. Effect of feeding systems, slaughter weight and sex on organoleptic properties and fatty acid composition of lamb. Journal of Animal Science, Champaign, v. 51, n. 2, p. 321-330, Aug. 1981.

KEY, A.; ANDERSON, J. T.; GRANDE, F. Serum cholesterol response to changes in the diet: IV. particular satured fatty acids in the diet. Metabolism, [S.1.], v. 14, p. 776-780, 1965.

MANCINI-FILHO, J.; CHEMIN, S. Aplicações nutricionais dos ácidos graxos trans. Óleos e grãos, São Caetano do Sul, v. 31, n. 1, p. 41-45, 1996. 
NAUGHTON, J. M.; O'DEA, K.; SINCLAIR, A. J. Animal foods in tradicional aboriginal diets: polyunsaturated and low in fat. Lipids, Champaign, v. 21, p. 684-690, 1986.

ODA, S. H. I. Diferentes métodos de abate e sexo na qualidade da carne de Capivara (Hydrochaeris hidrochaeris L. 1766). 2002. 145 p. Dissertação (Mestrado em Ciência dos Alimentos) - Universidade Federal de Lavras, Lavras, 2002.

OJASTI, J. Estudio biológico del chigüire o capibara. Caracas: FONAIAP, 1973.

ONYANGO, C. A.; IZUMIMOTO, M.; KUTIMA, P. $\mathrm{M}$. Comparison of some physical and chemical properties of seletedgame meats. Meat Science, Oxford, v. 49, n. 1, p. 117-125, May 1998.

PALEARI, M. A. et al. Ostrich meat physico chemical characteristics and comparison with turkey and bovine meat. Meat Science, Oxford, v. 48, n. 3/4, p. 205-210, Mar./Apr. 1998.

PARDI, M. C. et al. Ciência, higiene e tecnologia da carne: tecnologia da sua obtenção e transformação. Goiânia: Universidade de Goiás, 1993. v. 1, 586 p.

PEREZ, J. R. O. et al. Efeito do peso ao abate de cordeiros Santa Inês e Bergamácia sobre o perfil de ácidos graxos, colesterol e propriedades químicas. Ciência e Tecnologia de Alimentos, Campinas, v. 22, n. 1, p. 11-18, 2002.

RHEE, K. S. et al. Fatty acid compositin of goat diets vc intramuscular fat. Meat Sciense, Oxford, v. 54, n. 4, p. 313-318, Apr. 2000.

ROÇA, R. O. et al. Desenvolvimento de produtos curados e defumados com carne de capivara (Hydrochoerus hydrochaeris). In: CONGRESSO BRASILEIRO DE CIÊNCIA E TECNOLOGIA DE ALIMENTOS, 15., 1996, Poços de Caldas. Anais... Poços de Caldas: SBCTA, 1996.

ROSA, F. C. Teor de ácidos graxos poliinsaturados ômega-3 no peito e na coxa de frangos de corte alimentados com rações contendo três fontes de óleo. 1999. 93 p. Dissertação (Mestrado em Zootecnia) Universidade Federal de Lavras, Lavras, 1999.

ROSTAGNO, H. S. et al. Composição de alimentos e exigências nutricionais de aves e suínos: tabelas brasileiras. Viçosa: UFV, 2000. 141 p.
ROWE, A. et al. Muscle composition and fatty acid profile in lambs fattened in drylot or pasture. Meat Sciense, Oxford, v. 51, n. 4, p. 283-388, Apr. 1999.

SALDANHA, T.; SANTANA, D. M. N.; GASPAR, A. Lipídeos totais, colesterol e composição de ácidos graxos da carne de capivara (Hydrochoerus hydrochaeris). Brazilian Journal Of Food Technology, Campinas, v. 105, p. 245-149, 2002.

SALES, J. et al. Cholesterol content and fatty acid composition of rhea meat. Meat Science, Oxford, v. 53, p. 73-75, 1999.

SANTOS, C. L. Estudo do desempenho, das características da carcaça e do crescimento alométrico de cordeiros das raças Santa Inês e Bergamácia. 1999. 143 p. Dissertação (Mestrado em Zootecnia) - Universidade Federal de Lavras, Lavras, 1999.

SINCLAIR, A. J.; O'DEA, K. Fats in Human diets through history: is the western diet out of step? In: WOOD, J. D.; FISHER, A. V. Reducing fat in meat animals. London: Elsevier, 1990. p. 1-47.

SINCLAIR, A. J.; SLATTERY, W. J.; O'DEA, K. The analysis of poliunsaturated fatty acids in meat by capillary gas-liquid chromatography. Journal Science Food Agriculture, London, v. 33, n. 8, p. 771-776, Aug. 1982.

SOUZA, X. R. et al. Composição centesimal do músculo Biceps femoris de cordeiros em crescimento. Ciência e Agrotecnologia, Lavras, p. 1507-1513, dez. 2002.

SPECTOR, A. A. Essentialy of fatty acids. Lipids, Champaign, v. 34, p. S1-S3, 1999.

WALDMAN, R. C.; SUESS, G. C.; BRUNGARDT, V. H. Fatty acid composition tissue and their association with growth carcass and palatability traits. Journal of Animal Sciense, Champaign, v. 27, n. 3, p. 632-635, Aug. 1965.

WOOD, J. D. Consequences for meat quality of reducing carcass fatness. In: WOOD, J. D.; FISHER, A. $\mathrm{V}$. Reducing fat in meat animals. London: Elsevier, 1990. p. 344-389. 